\title{
Emergence of Carbapenem resistant Gram negative and vancomycin resistant Gram positive organisms in bacteremic isolates of febrile neutropenic patients: A descriptive study
} Seema Irfan*, Faiza Idrees, Vikram Mehraj, Faizah Habib, Salman Adil and Rumina Hasan

Address: Department of Pathology \& Microbiology, The Aga Khan University, Karachi, Pakistan

Email: Seema Irfan* - seema.irfan@aku.edu; Faiza Idrees - doctorfaiza@hotmail.com; Vikram Mehraj - vikram.mehraj@aku.edu; Faizah Habib - doc_faizah@hotmail.com; Salman Adil - salman.adil@aku.edu; Rumina Hasan - ruminahasan@gmail.com

* Corresponding author

Published: 9 June 2008

BMC Infectious Diseases 2008, 8:80 doi:10.1 |86/147| -2334-8-80

This article is available from: http://www.biomedcentral.com/I47I-2334/8/80

(c) 2008 Irfan et al; licensee BioMed Central Ltd.

This is an Open Access article distributed under the terms of the Creative Commons Attribution License (http://creativecommons.org/licenses/by/2.0), which permits unrestricted use, distribution, and reproduction in any medium, provided the original work is properly cited.
Received: 2 January 2008

Accepted: 9 June 2008

\begin{abstract}
Background: This study was conducted to evaluate drug resistance amongst bacteremic isolates of febrile neutropenic patients with particular emphasis on emergence of carbapenem resistant Gram negative bacteria and vancomycin resistant Enterococcus species.

Methods: A descriptive study was performed by reviewing the blood culture reports from febrile neutropenic patients during the two study periods i.e., 1999-00 and 200I-06. Blood cultures were performed using BACTEC 9240 automated system. Isolates were identified and antibiotic sensitivities were done using standard microbiological procedures.

Results: Seven twenty six febrile neutropenic patients were admitted during the study period. A total of 5840 blood cultures were received, off these 1048 ( $18 \%$ ) were culture positive. Amongst these, 557 (53\%) grew Gram positive bacteria, 442 (42\%) grew Gram negative bacteria, 43 (4\%) fungi and $6(1 \%)$ anaerobes. Sixty $(5.7 \%)$ out of 1048 positive blood cultures were polymicrobial. In the Gram negative bacteria, Enterobacteriaceae was the predominant group; $E$. coli was the most frequently isolated organism in both study periods. Amongst non- Enterobacteriaceae group, Pseudomonas aeruginosa was the commonest organism isolated during first study period followed by Acinetobacter spp. However, during the second period Acinetobacter species was the most frequent pathogen.

Enterobacteriaceae group showed higher statistically significant resistance in the second study period against ceftriaxone, quinolone and piperacillin/tazobactam, whilst no resistance observed against imipenem/meropenem. The susceptibility pattern of Acinetobacter species shifted from sensitive to highly resistant one with significant $p$ values against ceftriaxone, quinolone, piperacillin/tazobactam and imipenem/meropenem. Amongst Gram positive bacteria, MRSA isolation rate remained static, vancomycin resistant Enterococcus species emerged in second study period while no Staphylococcus species resistant to vancomycin was noted.
\end{abstract}

Conclusion: This rising trend of highly resistant organisms stresses the increasing importance of continuous surveillance system and stewardship of antibiotics as strategies in the overall management of patients with febrile neutropenia. 


\section{Background}

Febrile neutropenia is associated with high mortality rate therefore institution of timely and appropriate empirical antibiotic therapy is absolutely essential $[1,2]$. Globally significant change in the spectrum of organisms and their susceptibility pattern is observed in febrile neutropenic over the past few decades. Staphylococcus aureus was the most frequent isolate from these patients in 1950s and early 1960s and was later replaced by Gram-negative bacilli including Escherichia coli, Klebsiella species and Pseudomonas aeruginosa [3]. However since 1980s, resurgence of Gram-positive organisms in this population is evident [4]. Recently non-fermenter Gram negative rods such as Acinetobacter species have emerged as pathogens in these patients [5]. Additionally use of broad spectrum antibiotics has resulted in emergence of multi drug resistant Gram negative and gram positive bacteria. Therefore, the choice of empiric therapy should vary according to locally prevalent isolates and their resistance patterns. Currently, use of carbapenem as an empirical monotherapy for febrile neutropenic patient is justified in most centers due to growing resistance against other beta lactam antibiotics $[6,7]$. Similarly empirical use of vancomycin for suspected penicillin and methicillin resistant Gram positive bacterial infection is recommended [2].

Emergence and spread of carbapenem resistant Gram negative rods is a great concern, especially in a resource limited country such as Pakistan, where treatment alternatives are either unavailable or expensive/toxic with poor outcome [8]. The Aga Khan University is a major tertiary care hospital in Pakistan that caters a large population of severely immuno-suppressed patients. The hospital has a hematology-oncology unit along with a bone marrow transplant unit. Recent reports from our center have documented emergence and spread of carbapenem resistance among multi-resistant non enterobacteriaceae including Acinetobacter species and Pseudomonas aeruginosa $[9,10]$. In addition, our hospital infection control surveillance committee also reported rising trend of carbapenem resistance in Acinetobacter species and Pseudomonas aeruginosa, beginning from the year 2001. This has raised the concern about possibility of emergence of these pathogens in febrile neutropenic patients. Similarly rising trend of methicillin resistant Staphylococcus aureus and emergence of vancomycin resistant enterococci amongst the hospitalized patients are of great concern [11]. We therefore conducted a study to evaluate drug resistance in bacteremic isolates of febrile neutropenic patients with particular emphasis on emergence of carbapenem resistant gram negative bacteria. In addition to that we tried to evaluate the frequency of methicillin resistant Staphylococcus aureus and vancomycin resistance Enterococcus species isolated from blood cultures in these patients.

\section{Methods}

This study was conducted in the department of Pathology and Microbiology, Aga Khan University Karachi, Pakistan. Medical records and blood cultures of febrile neutropenic patients, admitted from year 1999 to 2006 were reviewed. To evaluate the changing pattern and increasing antimicrobial resistance of microorganisms in febrile neutropenic patients, we divided the study duration in two periods, the first period comprised of January 1999 to December 2000 while the second period was from January 2001 to December 2006.

Neutropenia was defined as neutrophil counts of 500 or 1000 with predicted decline to 500. Fever was defined as oral temperature of $38^{\circ} \mathrm{C}$ or above for at least one hour [1]. Blood cultures received from all febrile neutropenic patients between this six and half year study period were included in the study. To avoid error the duplicates were removed (we considered duplicate if same isolate grew more than once during that admission).

Blood cultures were performed using BACTEC 9240 automated system. A set of aerobic and anaerobic bottles containing brain heart infusion and thioglycolate broth respectively, were used for cultures. Negative blood culture bottles were incubated for seven days before being reporting negative. Blood culture isolates were identified using standard microbiological procedure and further confirmation was done using commercially available API strips [12]. Antibiotic sensitivities of all isolates except non enterobacteriaceae were carried out using Kirby Bauer method [13]. Sensitivity testing for non enterobacteriaceae was done using agar dilution method as recommended by CLSI [13].

Statistical analysis: Descriptive analysis was done by calculating frequency and percentages. In order to evaluate the difference in the sensitivity patterns of both gram negative and gram positive organisms during the two halves of the study period, chi square test or Fisher exact where appropriate was performed with a significance level of $5 \%$.

\section{Results}

During the study period (1999-2006), 726 febrile neutropenic patients were admitted. We reviewed medical records of randomly selected 134/726 (18.4\%) patients. Amongst these, $49 \%$ belonged to hematological malignancy, $44 \%$ had solid organ cancer while in $7 \%$ of cases diagnosis was not established. Chest was the commonest site of infection $(21 \%)$, followed by skin \& soft tissue $(12.6 \%)$, gastroenteritis $(11 \%)$, mucositis $(8.2 \%)$, porta cath $(8.2 \%)$ and others $(9.7 \%)$. No obvious focus of infection was found in $25.3 \%$ cases. 
A total of 5840 blood cultures were received, off these 1048 (18\%) were culture positive. Amongst these, 557 (53\%) grew Gram positive bacteria, 442 (42\%) grew Gram negative bacteria, $43(4 \%)$ fungi and $6(1 \%)$ anaerobes. Sixty out of 1048 (5.7\%) positive blood cultures were polymicrobial. Amongst these $93.4 \%$ were positive for two organisms, $5 \%$ for three and $1.6 \%$ for four organisms. Increasing trend was observed in the isolation rate of gram positive bacteria i.e. from $50 \%$ in first study period to $54 \%$ in the second one. A little drop in isolation rate of gram negative bacteria was noted i.e. from $43 \%$ to $41 \%$. No statistically significant difference for the isolation rate of Staphylococci, Streptococci and other gram positive bacteria was found between the two study periods, as shown in Table 1.

Amongst the gram negative bacteria, Enterobacteriaceae remained the predominant group, comprising of $65 \%$ and $64 \%$ of total gram negative bacteria in the first and second study period respectively as shown in Table 2. E. coli was the most frequently isolated organism in both study periods. The isolation frequency of non enterobacteriaceae was found to be static in both study periods i.e. $25-30 \%$ of the total gram negative organisms. Amongst this group, Pseudomonas aeruginosa was the commonest organism isolated during first study period followed by Acinetobacter spp. However, during the second period Acinetobacter species was the most frequent pathogen. Another important finding was the emergence of Stenotrophomonas maltophilia, Aeromonas species and Achromobacter species during the second study period.

A significant rise in drug resistant isolates was noted in the second study period. This increase was identified for all gram negative bacteria including Enterobacteriaceae, Pseudomonas aeruginosa, other Pseudomonas species and Acinetobacter species as shown in Table $3 \mathrm{a} \& 3 \mathrm{~b}$. Amongst the
Enterobacteriaceae, statistically significant resistance appeared in the second study period against ceftriaxone ( $\mathrm{p}$ $=0.001)$, ciprofloxacin/ofloxacin $(\mathrm{p}=0.003)$ and piperacillin/tazobactam $(\mathrm{p}=0.006)$. However no resistance against imipenem/meropenem was detected in second study period. Fifty four percent of Enterobacteriaceae were ESBL positive during the second study period.

Similarly increased resistance in Pseudomonas aeruginosa was noted against amikacin and ciprofloxacin/ofloxacin during the second study period. However, this difference was not statistically significant $(\mathrm{p}>0.05)$. No resistance against piperacillin/tazobactam and imipenem/meropenem was found during the first study half; however resistance against both of these antibiotics (6\%) appeared in the second study period. The susceptibility pattern of Acinetobacter species shifted from sensitive to highly resistant one with significant $p$ values against third generation cephalosporin $(\mathrm{p}=0.001)$, quinolone $(\mathrm{p}=0.004)$, piperacillin/tazobactam $(\mathrm{p}=0.003)$ and imipenem/meropenem $(\mathrm{p}=0.003)$. During the second study period $37 \%$ (49/134) of total non- enterobacteriaceae were found imipenem/meropenem resistant making a total of $14 \%$ (49/ 352 ) imipenem resistance amongst all gram negative bacteria.

Among the gram positive bacteria isolation frequency of methicillin resistant Staphylococcus aureus was not statistically significant ( $\mathrm{p}=0.524)$, however, vancomycin resistant Enterococcus species emerged in second half of study as shown in Table 4. No Staphylococcus species resistant to vancomycin was isolated.

\section{Discussion}

Our study showed rising trend of carbapenem resistant Gram negative and vancomycin resistant Gram positive bacteria among bacteremic isolates of febrile neutropenic

Table I: Gram positive bacteria isolated from blood cultures in two halves of study period.

\begin{tabular}{|c|c|c|c|c|c|}
\hline \multirow[b]{2}{*}{ Gram positive Bacteremic isolates } & \multicolumn{2}{|c|}{ (Period I) Positive blood culture } & \multicolumn{2}{|c|}{ (Period II) Positive blood culture } & \multirow[b]{2}{*}{ P-value } \\
\hline & $n=104$ & $\%$ & $n=453$ & $\%$ & \\
\hline Staphylococcus spp. & 56 & 53.8 & 250 & 55.2 & 0.804 \\
\hline Staphylococcus aureus & 14 & 13.5 & 43 & 9.5 & 0.228 \\
\hline Streptococcus spp. & 6 & 5.8 & 25 & 5.5 & 0.920 \\
\hline Enterococcus spp. & 7 & 6.7 & 23 & 5.1 & 0.501 \\
\hline Bacillus spp. & 10 & 9.6 & 44 & 9.7 & 0.976 \\
\hline Micrococcus spp. & 3 & 2.9 & 17 & 3.8 & $0.999 \#$ \\
\hline Streptococcus pneumoniae & 2 & 1.9 & 16 & 3.5 & $0.548 \#$ \\
\hline Corynebacterium spp. & 5 & 4.8 & 25 & 5.5 & 0.772 \\
\hline Streptococcus pyogenes & I & 1.0 & 5 & I.I & $0.999 \#$ \\
\hline Nocardia spp. & 0 & 0.0 & I & 0.2 & $0.999 \#$ \\
\hline Other Gram positive organism & 0 & 0.0 & 4 & 0.9 & $0.999 \#$ \\
\hline
\end{tabular}

$\mathrm{p}$-values calculated by Chi square test otherwise indicated

\# p-value calculated by Fisher exact test 
Table 2: Gram negative bacteria isolated from blood cultures in two halves of study period

\begin{tabular}{|c|c|c|c|c|c|}
\hline \multirow[b]{2}{*}{ Gram Positive Bacteremic Isolates } & \multicolumn{2}{|c|}{ (Period I) Positive blood culture } & \multicolumn{2}{|c|}{ (Period II) Positive blood culture } & \multirow[b]{2}{*}{ P-value } \\
\hline & $\mathrm{n}=90$ & $\%$ & $n=352$ & $\%$ & \\
\hline E. coli & 26 & 28.9 & 129 & 36.6 & 0.169 \\
\hline$P$. aeruginosa & 15 & 16.7 & 34 & 9.7 & 0.059 \\
\hline Enterobacter spp. & 12 & 13.3 & 30 & 8.5 & 0.165 \\
\hline Pseudomonas spp. & 8 & 8.9 & 29 & 8.2 & 0.842 \\
\hline Klebsiella spp. & 11 & 12.2 & 41 & 11.6 & 0.880 \\
\hline Acinetobacter spp. & 6 & 6.7 & 52 & 14.8 & $0.042 *$ \\
\hline Salmonella & 4 & 4.4 & 12 & 3.4 & $\left.0.75\right|^{\#}$ \\
\hline Citrobacter spp. & 3 & 3.3 & 6 & 1.7 & $0.396 \#$ \\
\hline Proteus spp. & 3 & 3.3 & I & 0.3 & 0.028 \#* \\
\hline Aeromonas spp. & 1 & 1.1 & 8 & 2.3 & $0.693 \#$ \\
\hline Stenotrophomonas maltophilia & 0 & 0.0 & 7 & 2.0 & $0.353 \#$ \\
\hline Achromobacter spp & 0 & 0.0 & I & 0.3 & $0.999 \#$ \\
\hline Alcaligenes xylosoxidans & I & 1.1 & 0 & 0.0 & $0.204 \#$ \\
\hline Chryseomonas luteola & 0 & 0.0 & 1 & 0.3 & $0.999 \#$ \\
\hline Campylobacter spp. & 0 & 0.0 & I & 0.3 & $0.999 \#$ \\
\hline
\end{tabular}

$\mathrm{P}$-values calculated by Chi square test otherwise indicated \# p-value calculated by Fisher exact test

$* p<0.05$

patients. The emergence of carbapenem resistant isolates in our center is likely to be associated with change in antibiotic policies. The two study periods were chosen based on difference in empirical antibiotic choice in our center. During nineties third generation cephalosporin was the empirical antibiotic choice for febrile neutropenic patients at our institute [14]. However, rising trend of resistance against this group of antibiotics was observed among Enterobacteriaceae and Pseudomonas aeruginosa $[15,16]$. Prior to year 2000, there was limited availability of piperacillin/tazobactam and imipenem. These agents were introduced throughout the hospital in year 2000 [15].

Our study revealed no resistance to carbapenem and minimal resistance against piperacillin/tazobactam in Gram negative isolates during the first study period. However, rising trend of resistance against these agents was noted in second period. This change was specific for non enterobacteriaceae group. These bacteria showed significant rise in resistance against major antibiotics including third generation cephalosporin, quinolone, amikacin, piperacillin/ tazobactam and carbapenem. In contrast to this no resistance against carbapenem was noted amongst Enterobacteriaceae group.

Another important finding was increased isolation rates of Acinetobacter species. This organism was the sixth Gram negative bacteria isolated during the first study period; however it became the second most commonly isolated gram negative bacteria during the second study period. Moreover, carbapenem resistance in this bacterium was significantly high $(\mathrm{p}=0.003)$ during second study period, indicating possible role of nosocomial transmission for this rising trend. Finally, emergence of Gram negative organism inherently resistant to carbapenem like Stenotrophomonas maltophilia during the second study period was another area of concern, a finding consistent with other reports [17]. Our findings are in contrast to other regional reports, where carbapenem resistance in this population is not observed $[18,19]$.

The isolation rate of MRSA during the two study halves was comparable $(\mathrm{p}=0.524)$. The isolation rate of MRSA (31\%) was significantly high when compared with the previous study done in 1991 which reported 100\% susceptibility against cloxacillin [14]. Similar increase in isolation rate of methicillin resistant Staphylococcus aureus (MRSA) was also reported in another study from Taiwan [20]. Moreover emergence of vancomycin resistant Enterococcus species (VRE) not only poses a therapeutic challenge for febrile neutropenic patients $[21,22]$ but also indirectly reflects irrational usage of vancomycin and poor infection control practices. The increasing rates of antimicrobial resistance amongst both Gram-positive and Gramnegative pathogens isolated from patients with neutropenia are posturing new therapeutic challenges. These challenges are compounded by the fact that relatively few new drugs are being developed, particularly those that treat resistant Gram-negative organisms [22]. As these trends are often associated with local treatment practices $[22,23]$ therefore, we suggest rational use of broad-spectrum antibiotics especially carbapenem and vancomycin to prevent increasing resistance against them. In addition compro- 
Table 3: Difference in sensitivity pattern of frequently isolated Gram negative rods during two halves of study period.

(a)

\begin{tabular}{|c|c|c|c|c|c|c|c|c|c|c|}
\hline & \multicolumn{5}{|c|}{ Enterobacteriaceae } & \multicolumn{5}{|c|}{ Pseudomonas. aeruginosa } \\
\hline & \multicolumn{2}{|c|}{ Period I } & \multicolumn{2}{|c|}{ Period II } & \multirow[b]{2}{*}{ P-value } & \multicolumn{2}{|c|}{ Period I } & \multicolumn{2}{|c|}{ Period II } & \multirow[b]{2}{*}{ P-value } \\
\hline & $n=55$ & $\%$ & $n=212$ & $\%$ & & $n=15$ & $\%$ & $\mathrm{n}=\mathbf{3 4}$ & $\%$ & \\
\hline Amikacin & 6 & 10.9 & 35 & 16.5 & 0.305 & 0 & 0 & 4 & 11.8 & $0.298 \#$ \\
\hline Amox/clav & 25 & 45.5 & 102 & 48.1 & 0.725 & NT & NT & NT & NT & - \\
\hline Ceftriaxone & 13 & 23.6 & 118 & 55.7 & $<0001 *$ & NT & NT & NT & NT & - \\
\hline Ciprofloxacin & 15 & 27.3 & 105 & 49.5 & $0.003 *$ & 2 & 13.3 & 10 & 29.4 & $0.298 \#$ \\
\hline Tazocin & 0 & 0 & 24 & 11.3 & $0.006 *$ & 0 & 0 & 4 & 11.8 & 0.298 \# \\
\hline Imipenem & 0 & 0 & 0 & 0 & - & 0 & 0 & 2 & 5.9 & $0.999 \#$ \\
\hline Ceftazidime & NT & NT & NT & NT & - & I & 6.7 & 2 & 5.9 & $0.999 \#$ \\
\hline
\end{tabular}

(b)

\begin{tabular}{|c|c|c|c|c|c|c|c|c|c|c|}
\hline & \multicolumn{5}{|c|}{ Pseudomonas species } & \multicolumn{5}{|c|}{ Acinetobactor } \\
\hline & \multicolumn{2}{|c|}{ Period I } & \multicolumn{2}{|c|}{ Period II } & \multirow[b]{2}{*}{ p-value } & \multicolumn{2}{|c|}{ Period I } & \multicolumn{2}{|c|}{ Period II } & \multirow[b]{2}{*}{ p-value } \\
\hline & $n=8$ & $\%$ & $n=29$ & $\%$ & & $n=6$ & $\%$ & $n=52$ & $\%$ & \\
\hline Amikacin & 4 & 50.0 & 12 & 41.4 & $0.705 \#$ & 1 & 16.7 & 27 & 51.9 & $0.195 \#$ \\
\hline Amox/clavilunate & NT & NT & NT & NT & - & NT & NT & NT & NT & - \\
\hline Ceftriaxone & 0 & 0 & 7 & 24.1 & $0.308 \#$ & 0 & 0 & 39 & 75.0 & $0.001 \# *$ \\
\hline Ciprofloxacin & 1 & 12.5 & 7 & 24.1 & $0.655 \#$ & 0 & 0 & 33 & 63.5 & $0.004 \# *$ \\
\hline Pipracillin/Tazobactam & 0 & 0 & 2 & 6.9 & $0.999 \#$ & 0 & 0 & 34 & 65.4 & $0.003 \#^{*}$ \\
\hline Imipenem & 0 & 0 & 6 & 20.7 & $0.305 \#$ & 0 & 0 & 34 & 65.4 & $0.003 \# *$ \\
\hline Ceftazidime & NT & NT & NT & NT & - & NT & NT & NT & NT & - \\
\hline
\end{tabular}

p-values calculated by Chi square test otherwise indicated \# p-value calculated by Fisher exact test

$* p<0.05$

Table 4: Difference in sensitivity pattern of frequently isolated Gram positive bacteria during two halves of study period.

\begin{tabular}{|c|c|c|c|c|c|c|c|c|c|c|c|c|c|c|c|}
\hline & \multicolumn{4}{|c|}{$\begin{array}{c}\text { Staphylococcus species other than } \\
\text { aureus }\end{array}$} & \multicolumn{6}{|c|}{ Staphylococcus aureus } & \multicolumn{5}{|c|}{ Enterococcus species } \\
\hline & \multicolumn{2}{|c|}{ Period I } & \multicolumn{2}{|c|}{ Period II } & \multirow[b]{2}{*}{ p-value } & \multicolumn{2}{|c|}{ Period I } & \multicolumn{2}{|c|}{ Period II } & \multirow[b]{2}{*}{ p-value } & \multicolumn{2}{|c|}{ Period I } & \multicolumn{2}{|c|}{ Period II } & \multirow[b]{2}{*}{ p-value } \\
\hline & $n=56$ & $\%$ & $n=250$ & $\%$ & & $n=14$ & $\%$ & $n=43$ & $\%$ & & $n=7$ & $\%$ & $n=23$ & $\%$ & \\
\hline $\begin{array}{l}\text { Vancom } \\
\text { ycin }\end{array}$ & 0 & 0 & 0 & 0 & - & 0 & 0 & 0 & 0 & - & 0 & 0 & 3 & 13.0 & $0.999 \#$ \\
\hline $\begin{array}{l}\text { Cloxacill } \\
\text { in }\end{array}$ & 30 & 53.6 & 130 & 52.0 & 0.831 & 3 & 21.4 & 13 & 30.2 & $0.735 \#$ & NT & - & NT & - & - \\
\hline Penicillin & 49 & 87.5 & 192 & 76.8 & 0.077 & 12 & 85.7 & 40 & 93.0 & $0.587 \#$ & NT & - & NT & - & - \\
\hline $\begin{array}{l}\text { Clindam } \\
\text { ycin }\end{array}$ & 20 & 35.7 & 82 & 32.8 & 0.676 & 3 & 21.4 & 13 & 30.2 & $0.735 \#$ & NT & - & NT & - & - \\
\hline Ampicilli & NT & - & NT & - & - & NT & - & - & NT & - & 7 & 100 & 17 & 73.9 & $0.290 \#$ \\
\hline
\end{tabular}

p-values calculated by Chi square test otherwise indicated

\# p-value calculated by Fisher exact test 
mised infection control practice is another contributing factor, hence early detection and prompt isolation of patients with strict compliance to hand hygiene is important to prevent further spread of multi resistant organisms.

\section{Conclusion}

This rising trend of highly resistant organisms stresses the increasing importance of continuous surveillance system and stewardship of antibiotics as strategies in the overall management of patients with febrile neutropenia.

\section{Competing interests}

The authors declare that they have no competing interests.

\section{Authors' contributions}

SI conceived, planned, conducted and generated results of the study, and drafted the manuscript. FI performed data collection and analysis and contributed in manuscript writing. FH and VM performed the statistical analysis and contributed in manuscript writing. SA contributed in provision of clinical information and approved the final manuscript. RH participated in study design and coordination and had contributed in manuscript writing and final approval of script. All authors read and approved the final manuscript.

\section{Acknowledgements}

This study was supported through a grant from the Joint Pakistan-US Academic and Research Program HEC/MoST/USAID. We would like to thank Dr Mohammad Khurshid, Dr Kauser Jabeen, all faculty and staff of the Clinical Microbiology Laboratory of the Aga Khan University Hospital, Karachi for their support and help in this study.

\section{References}

I. Glasmacher A, Von Lilienfeld-Toal M, Schulte S, Hahn C, SchmidtWolf IG, Prentice A: An evidence-based evaluation of important aspects of empirical antibiotic therapy in febrile neutropenic patients. Clin Microbiol Infect 2005, I I: I 7-23.

2. Walter Hughes T, Dowald Armtrong, Genald Bodey P, Eric Bow J, Arthur Brown E, Thierry Calandra, et al:: Guidelines for the use of antimicrobial agents in neutropenic patients with cancer. Clin Infect Dis 2002, 34:730-748.

3. Jones RN: Contemporary antimicrobial susceptibility pattern of bacterial pathogens commonly associated with febrile patients with neutropenia. Clin Infect Dis 1999, 29:495-502.

4. Sharma A, Lokeshwar N: Febrile neutropenia in haematological malignancies. J Postgrad Med 2005, 5 I :42-48.

5. Wojak I, Gospodarek E: Analysis of microorganisms isolated from febrile neutropenic children with neoplastic disease. Med Dosw Mikrobiol 2004, 56:4I I-9.

6. Ramphal R: Changes in the etiology of bacteremia in febrile neutropenic patients and the susceptibilities of the currently isolated pathogens. Clin Infect Dis 2004:25-3I.

7. Paul M, Yahav D, Fraser A, Leibvici L: Empirical antibiotic monotherapy for febrile neutropenia: systematic review and met analysis of randomized control trials. J Antimicrob Chemother 2006, 57:176-189.

8. Hirakata $Y$, Yamaguchi T, Nakano M, Izumikawa K, Mine M, Aoki S, Kondoh A, Matsuda J, Hirayama M, Yanagihara K, Miyazaki Y, Tomono K, Yamada Y, Kamihira S, Kohno S: Clinical and bacteriological charachteristics of IPM- type metallo- $\beta$-lactamase producing Pseudomonas aeruginosa. Clin Infect Dis 2003, 37:26-32.
9. Sarwari A, Hasan $\mathrm{R}, \operatorname{Lim} \mathrm{C}, \mathrm{Ng} \mathrm{Y}, \mathrm{Ng} C$, Zaman S: PCR identification and automated ribotyping of Pseudomonas aeruginosa clinical isolates from intensive care patients. Scandininavian J Infect Dis 2004, 36:342-349.

10. Noor A, Hussain SF: Risk factors associated with development of ventilator associated pneumonia. JCPSP 2005, I 5(2):92-95.

II. Khan E, Sarwari A, Hasan R, Ghori S, Babar I, O'Brien F, Grubb W: Emergence of vancomycin resistant Enterococcus Faeium at $a$ tertiary care hospital in Karachi, Pakistan. J Hospital Infection 2002, 52:292-296.

12. Koneman EW, Allen SD, Janda WM, Scherckenberger PC, Winn JWC: Color atlas and textbook of diagnostic microbiology. 6th edition. Philadelphia Lippincott; 2002.

13. National Committee for Clinical Laboratory Standards: Performance standards for antimicrobial susceptibility testing. $10^{\text {th }}$ informational supplement. Edited by: Wayne PA. National Committee for Clinical Laboratory Standards; 2004.

14. Karim M, Khan W, Faroogi B, Malik I: Bacterial isolates in neutropenic febrile patients. J Pak Med Assoc I99I, 48:364-7.

15. Khan MA, Siddiqui BK, Shamim A, Yosuf MA, Ahmed A, Zakiullah N, Burney IA: Emerging bacterial resistance pattern in febrile neutropenic patients: experience at atertiary care hospital in Pakistan. J Pak Med Assoc 2004, 54:357-360.

16. Burney IA, Farooqui BJ, Siddiqui T, Khurshid M: The spectrum of bacterial infections in febrile neutropenic patients. The effect on empiric antibiotic therapy. J Pak Med Assoc 1998, 48( I 2):364-7.

17. Gales AC, Jones RN, Forward KR, Linares J, Sader HS, Verhoef J: Emerging importance of multi-drug resistant Acinetobacter species and Stenotrophomonas maltophilia as pathogens in seriously ill patients: geographic patterns, epidemiological features, and trends in the SENTRY antimicrobial surveillance program (1997-1999). Clin Infect Dis 200I, 32:SI04-I3.

18. Baskaran ND, Gan GG, Adeeba K, Sam IC: Bacteremia in patients with febrile neutropenia after chemotherapy at a university medical center in Malaysia. Int J Infect Dis 2007, I I:5 I3-7.

19. Wang FD, Lin ML, Liu CY: Bacteremia in patients with hematological malignencies. Chemotherapy 2005, 5 I : | 47-53.

20. Chen CY, Tang JL, Hsueh PR, Yao M, Huang SY, Chen YC, Shen MC, Wang $\mathrm{CH}$, Tsai W, Chang SC, Tien HF, Luh KT: Trends and antimicrobial resistance of pathogens causing bloodstream infections among febrile neutropenic adults with hematological malignancy. J Formos Med Assoc 2004, $103(7): 526-32$.

21. Eil-Mahallawy H, Sidhom I, El-Din NH, Zamzam M, El-Lamie MM: Clinical and microbiological determinants of serious blood stream infections in Egyptian pediatric patients; One year study. Int J Infect Dis 2005, 9(I):43-5I.

22. Kline RM, Baorto EP: Treatment of pediatric febrile neutropenia in the era of vancomycin-resistant microbes. Pediatr Blood Cancer 2005, 44:205-6.

23. Feld R: Vancomycin as a part of initial empirical antibiotic therapy for febrile neutropenia in patients with cancers: Pros and cons. Clin Infect Dis 1999, 29:503-7.

\section{Pre-publication history}

The pre-publication history for this paper can be accessed here:

http://www.biomedcentral.com/1471-2334/8/80/prepub 